\title{
1 Ultrahigh-Throughput ESI-MS: Sampling pushed to 2 six Samples per Second by Acoustic Ejection Mass Spectrometry
}

\section{Tim T. Häbe ${ }^{1 *}$, Chang Liu ${ }^{2}$, Tom R. Covey ${ }^{2}$, Roman P. Simon ${ }^{1}$, Wolfgang Reindl ${ }^{1}$, Frank H.} Büttner ${ }^{l}$, Martin Winter ${ }^{1}$, Daniel Bischoff ${ }^{l}$, Andreas H. Luippold ${ }^{1}$, and Frank Runge ${ }^{1 *}$

\author{
${ }^{1}$ Boehringer Ingelheim Pharma GmbH \& Co. KG, Drug Discovery Sciences, Birkendorfer \\ Strasse 65, 88397 Biberach an der Riss, Germany \\ ${ }^{2}$ SCIEX, 71 Four Valley Drive, Concord, Ontario L4K 4V8, Canada
}

*Correspondence to:

Tim T. Häbe, Tim.Haebe@Boehringer-Ingelheim.com

Frank Runge, Frank.Runge@Boehringer-Ingelheim.com

\begin{abstract}
We present an acoustic ejection mass spectrometry (AEMS) setup for ESI-MS based sample injection at a sampling rate faster than current ESI and MALDI techniques. A modified acoustic droplet ejection system was combined with an open port interface and a modified ESI source. To simulate applications of drug metabolism and pharmacokinetics analysis and high-throughput screening campaigns, two stress tests were performed regarding ion suppression and system endurance in combination with minor sample preparation. Maximum sampling rate was $6 \mathrm{~Hz}$ for dextromethorphan and $d_{3}$-dextrorphan (each $100 \mathrm{nM}$ ) for 1152 injections in $63 \mathrm{~s}$ at FWHM of $105 \mathrm{~ms}$ and \%RSD of 7.7\%/7.5\% without internal standard correction. Enzyme assay buffer and crude dog plasma caused signal suppression of $51 \% / 73 \%$ at $\% R S D$ of $5.7 \% / 6.7 \%(n=120)$ and stable OPI performance during 1100 injections. An endurance buffer revealed minor OPI pollution and constant signals for $>25.000$ injections $(\% R S D=8.5 \%, n=10,557)$.
\end{abstract}




\section{Introduction}

Electrospray ionization based mass spectrometry (ESI-MS) applications are still inadequate for routine high-throughput (HT) analysis as they are not as time efficient as chemiluminescence- or fluorescence assays. ${ }^{[1]}$ The limitations of these assays regarding labeled substrates, fluorescence artefacts, unphysiological conditions, limited dynamic range, specific antibodies and other bias in combination with the lack of mass selective quantitation or options for structure elucidation, underscore the need for rapid MS techniques. The performance of matrix-assisted laser desorption/ionization-time-of-flight (MALDI-TOF) MS in combination with automated target plate preparation has been demonstrated to be comparable to conventional HT assays (<1 s/sample). ${ }^{[2]}$ A label-free MALDI-TOF-based screening platform has been introduced recently for the identification of inhibitors of human cyclic GMP-AMP synthase. ${ }^{[3,4]}$ Results of a library screen of more than 1 million diverse small molecules demonstrated its suitability for large-scale HT screening readouts and a MALDI-TOF sample throughput of 0.4 s/sample, pushing the speed-limiting step back to the biochemical assay. ${ }^{[4,5]}$

The inherent caveat of the decoupled sample preparation and analysis steps in MALDI compared to electrospray ionization (ESI), drives the need for an ESI-HT-MS platform with equivalent throughput and readout quality. ESI-MS can cover a larger analyte/target space and assay-scope of drug discovery, small scale reaction monitoring and other applications with high-throughput demands, using unlabeled compound at analysis conditions closer to the real physiological conditions. ${ }^{[6]}$

Currently, the fastest ESI-MS techniques is RapidFire (RF) MS yielding medium throughput sample cycles of 7-13 s with an intermediate solid-phase enrichment/cleanup step, ${ }^{[7-10]}$ as well as $2.5 \mathrm{~s}$ using a direct injection in the so-called BLAZE-mode. ${ }^{[11]}$ These approaches are widely used for label-free assays during profiling of drug potencies ${ }^{[12-15]}$ and profiling of compounds towards their ADME (absorption, distribution, metabolism, excretion) properties. ${ }^{[7-9]}$ It has been implemented in a fully automated Rapid and Integrated Analysis System (RIAS) to handle the entire analytical process of in vitro ADME assays with RF sampling cycles of $8 \mathrm{~s} .{ }^{[7,8]}$

Recently, a novel sampling technique by acoustic mist ejection was combined with an instant gas phase transfer-based MS technique as acoustic mist ionization (AMI). ${ }^{[16,17]}$ In parallel, the combination of acoustic droplet ejection (ADE) and ESI-MS via open port interface (OPI) emerged as a trendsetting advance. Different from the AMI technology with the generation of a plume of mist for each ejection event, the ADE approach generates a single precisely controlled nano-liter sized sample droplet each time. Here, the ejected droplet is captured in the vortex of a continuous liquid flow, diluted and delivered to a standard ESI source. Based on these steps, OPI is aiming to overcome the limitations of typical continuous-flow autosamplers, thus decreasing sample cycle times significantly, and allowing a contactless sample introduction in the low nanoliter-range. ${ }^{[18]}$

The OPI is based on a prototype from Gary Van Berkel, initially described in 2002 as surface sampling probe, and as self-aspirating heated nebulizer probe for thin-layer chromatography (TLC) plates, other surfaces, and liquids. ${ }^{[19-22]}$ The liquid junction was used to elute substances out of the TLC substance zones and transferring them to the MS system. This approach did not prevail for TLC-MS and thus, the corresponding elution head-based approaches dominated further developments of high-performance (HP)TLC-MS and their automation. ${ }^{[23,24]}$ The geometry and orientation of the probe was further modified, and the term OPI was adopted alongside the term open-port probe (OPP) ${ }^{[25,26]}$ and open-port sampling interface (OPSI). ${ }^{[27]}$ These interfaces were used for the direct introduction of liquids, ${ }^{[26,28]}$ solid-phase microextraction (SPME) devices, ${ }^{[25,29]}$ samples via Immediate Drop On Demand Technology (I-DOT) and material from transmission geometry laser ablation into the carrier flow. ${ }^{[30,31]}$ The I-DOT-OPSI-ESI-MS approach enabled a sample introduction of $5 \mathrm{~s} /$ sample with baseline separation of signal peaks. ${ }^{\text {[30] }}$

Based on AMI, an ECHO 555 (Labcyte, San Jose, California, USA) liquid handler was used to inject nano-liters of sample as a plume of pico-liter mists via acoustic ejection into a heated tube 
with a reverse polarity electrode orifice at the tube entrance. This setup yielded a throughput of $0.3 \mathrm{~s} / \mathrm{sample}$ for multiple injections from a single well or for individual sample wells but without baseline separation. ${ }^{[16,17,32]}$ Sample throughput data for $0.45 \mathrm{~s} /$ sample was shown for individual wells with baseline separated MS signals. ${ }^{[17]}$ This approach was recently used to identify inhibitors of a human histone deacetylase at a throughput of 100,000 samples per day. ${ }^{[17]}$ However, this approach ejects a steady mist of droplets directly in the gas stream of a transfer capillary. Thus, in absence of any active self-cleaning of the droplet intake and any dilution effect into a carrier liquid, the risk of probe contamination and ion suppression effects is present. Additionally, this specific mist-ionization technique has limitations such as an individual ionization characteristic and interor intrawell signal deviations of $25 \%$ that can just be lowered to $3.5 \%$ when isotope labeled standards were used. ${ }^{[17]}$

Recent adaptations of OPI-ESI-MS used modified versions of the ATS-G4P (EDC Biosystems, Fremont, California, USA) or the ECHO 555 liquid handler, both equipped with an OPI (SCIEX, Concord, Canada). In both systems, the acoustic transducer ejects precise sample droplets of an accurate low nanoliter volume vertically out of 384- or 1536-well plates filled with only 1.5$50 \mu \mathrm{L}$ of sample liquid. After a three component alignment (transducer, sample well, and OPI), the source plate was moved below the OPI to eject samples from individual wells. Ejected droplets were captured into the vortex of the carrier flow directed towards the ESI source. The OPI geometry provides a coaxial liquid flow with liquid delivery around a capillary and liquid intake at the tip of this capillary, where a liquid vortex was formed. The liquid was aspirated into the capillary according to a Venturi effect provided by the special design of the electrospray ionization (ESI) source. These adaptations allowed sample cycles of up to $2 \mathrm{~Hz}$ and to detect signals at mean FWHM of $0.21 \mathrm{~s}^{[18]}$

The improvements presented herein describe a remarkably efficient and reproducible hyphenation of ADE-OPI to ESI-MS, using an integrated ATS-G4P system modified primarily to minimize the distance between the OPI and the ESI source. The liquid vortex carrier liquid delivery was achieved by a highly stable micro-annular gear pump system integrated in the ATS-G4P system. The present work also examines the performance of the carrier liquid pump systems, the influence of capillary length between OPI and ESI electrode, and that of all the modifications of the ATSG4P system, OPI and ESI source.

For the first time, we present the technical steps and 3D-printing resources needed to modify a standard ATS-G4P liquid handler to enable the ADE-OPI-ESI-MS feature, and to achieve a sampling rate of up to $6 \mathrm{~Hz}$ via multiplexing of two MRM transitions (for at least two analytes in different samples). Nevertheless, even the $3 \mathrm{~Hz}$ setup with an independent, serial sample injection (for the same analyte in every sample) revealed to be currently the fastest ESI-MS technique and even to be faster than current MALDI techniques under realistic HT screening conditions. ${ }^{[4,11,30]}$ To demonstrate the ability to inject samples without sample preparation and to reach HT preconditions, a stress test with crude plasma and enzyme assay buffer was performed for $>1,000$ injections as well as an endurance test to simulate HT screening conditions with $>25,000$ continuous injections.

\section{Results and Discussion}

\subsection{Injection and signal performance}

The sub-second, contactless ADE-OPI hyphenation to ESI-MS/MS was realized by appropriate modifications of an ATS-G4P coupled to an Optiflow Turbo V ESI source. The key focus was optimizing the fastest possible sample injection into the ESI source while maintaining high sensitivity and signal performance (baseline separation and suitable peak shape with low tailing). This was mainly dependent on the flow rate of the carrier liquid to avoid excessive axial diffusion of the injected nanoliter-sample droplet into the carrier liquid during transit. A too slow carrier liquid and high axial diffusion lead to broad peaks or even peak overlap, depending on the 
injection rate and capillary length. The carrier liquid flow rate at the tip of the OPI (controlled by the pump) had to be exactly balanced with the carrier liquid intake into the transfer capillary. Thus, the formation of a robust liquid vortex at high flowrates relied essentially on the suction force generated by the Venturi effect at the tip of the ESI sprayer. At a fixed sprayer and electrode geometry, the force of the Venturi effect was primarily dependent on the nebulizer gas flow (GS1), whereas its effectiveness was depending on the carrier liquid backpressure and thus, the transfer capillary geometry and the physical properties of the liquid carrier.

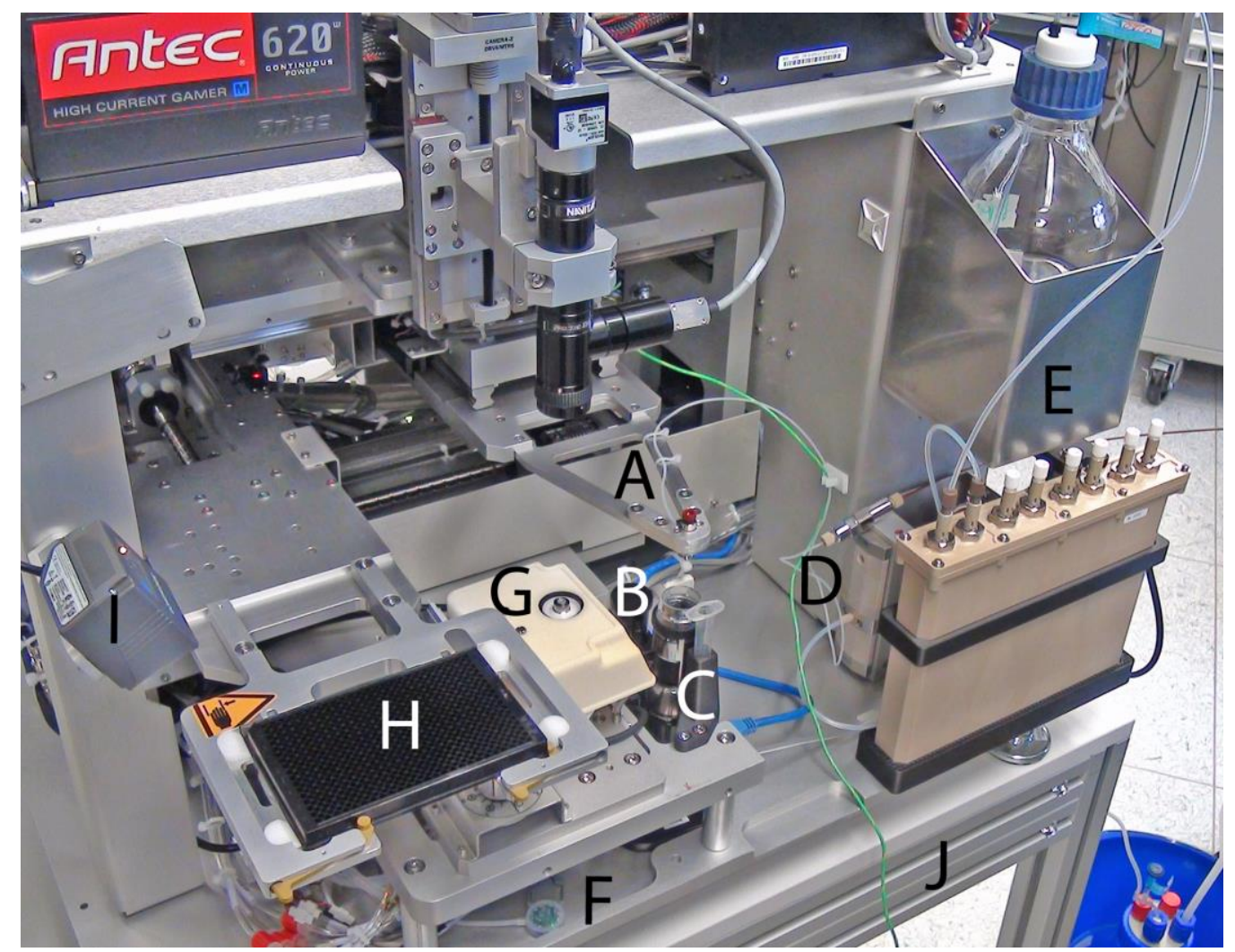

Figure 1 The modified EDC-G4P-based ADE-OPI setup: OPI mounted in its metal carrier extension (A) instead of the target plate carrier; microscope camera below the OPI (B); waste port (C); microgear pump (D) and the carrier liquid reservoir and degasser vacuum chamber (E); liquid sensor $(F)$ below the acoustic transducer $(\mathrm{G})$; 384-well plate in the source plate carrier at the new plate loading position $(\mathrm{H})$; barcode scanner (I). The whole system was placed at the correct height on a rack frame $(\mathrm{J})$

The Venturi effect occurs when the compressed nebulizer gas exits the ESI probe around the ESI electrode. The expanding gas produces a pressure drop in front of the electrode with a maximum force at an electrode protrusion of $0.5 \mathrm{~mm}$ out of the ESI probe. This pressure generates the suction force that pulled the carrier liquid through the transfer capillary (Figure S-6). In order to increase the pressure gradient even further (and hence the flow rate), the standard GS1 gas supply was increased to the maximum nitrogen flow rate of $19.5 \mathrm{~L} / \mathrm{min}$ trough the source tubing and the probe. To increase the effectiveness of the Venturi effect, the backpressure was reduced by shortening the ESI electrode and transfer capillary (at maximum ID) as much as possible. For even higher flow rates, carrier liquids with lower viscosity were investigated as well as the surface tension of the liquid inside the OPI. With this in mind, the first hurdle was therefore to minimize the distance between the OPI and the ESI electrode. The second hurdle was to find a suitable carrier liquid of low viscosity to maximize the efficiency of the modified OPI and ESI source for optimal sample injection speed and signal performance. 


\subsection{Basic hardware modifications}

The digitalization of the ATS-G4P, the Optiflow Turbo V source and the additional components enabled us to simulate the complete design and construction of additional components necessary to implement various modifications. This was crucial in order to optimize the shortest distance between the OPI and ESI electrode. The very first setup (Figure S-3A) using the unmodified instruments (Turbo V ESI source) placed on a standard working bench height $(\sim 78 \mathrm{~cm})$, revealed that an at least $60 \mathrm{~cm}$ long transfer capillary was necessary to connect the OPI with the ESI electrode. The instrument housings as well as the baseplate of the ATS-G4P were the main obstacle to bring both instruments closer. Thus, a rack frame (Figure 1J) was constructed for the ATS-G4P to level the OPI position with the capillary fitting of the ESI source electrode (Optiflow Turbo V). This shortened the capillary length and formed the basis for an integrated system providing all the hardware and software components to perform the OPI sample injection as well as data acquisition and evaluation of the MS system (Figure S-4).

For a short transfer capillary, the ESI source had to be aligned in the nearest position to the acoustic transducer. Thus, all unnecessary components of the ATS-G4P front end were removed or relocated (Figure S-2) and the instrument baseplate was cut directly in front of the mounting posts of the acoustic transducer unit (Figure S-8). These modifications enabled a shorter transfer capillary length of $30 \mathrm{~cm}$ and even a length of just $25 \mathrm{~cm}$ in combination with the subsequent modified ESI source (Figure S-3B).

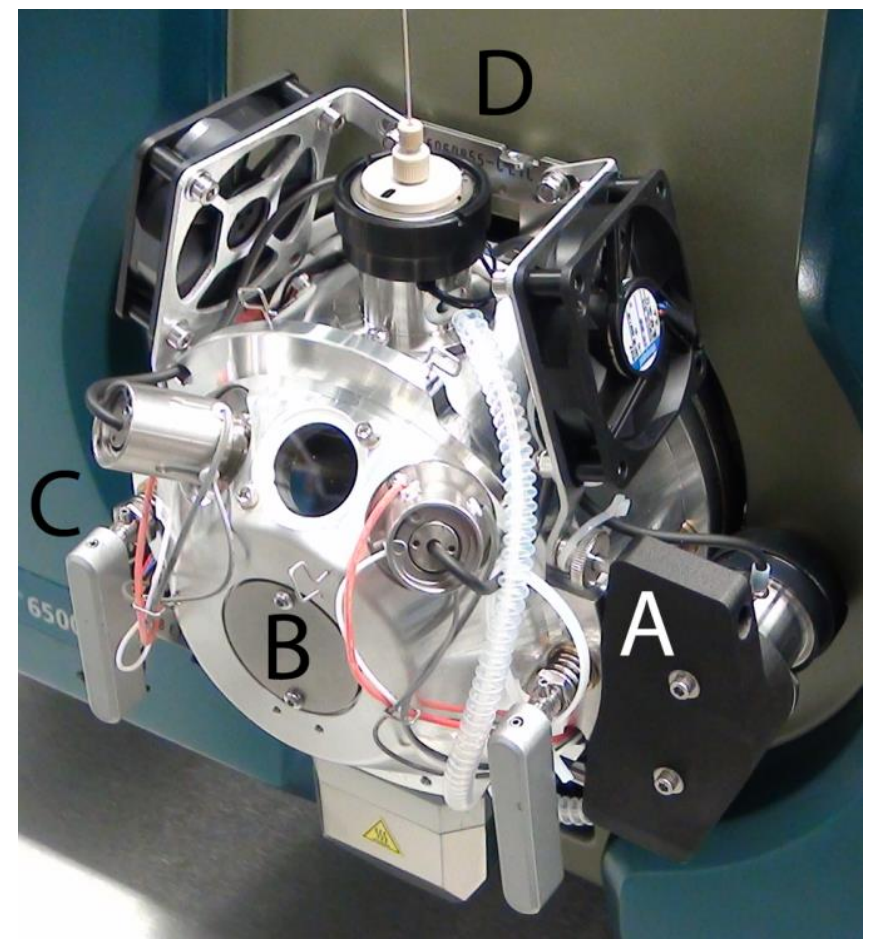

Figure 2. Modified ESI source for shortest OPI to ESI electrode distance (Figure S-3). The horizontal ESI sprayer was relocated in a holder on the right side of the source (A) and the source housing was sealed with a Teflon plate below a metal cover plate (B). The electronic board of the source was relocated on the left side ( $\mathrm{C}$, not visible) and the cooling fan of the original housing was replaced by two cooling fans directed between the source heaters and the ESI sprayer (D).

\subsection{Modifications on the ESI source} front was relocated to the right side of the source block (Figure 2A). As there was no additional source equipment required for this application, the high voltage switch was removed and its contact was closed on the electronic board. The board was relocated to the left side of the source on a new holder. As the cooling fan in the housing was removed and to provide a better heat 
dissipation between the source heaters and the sprayer flange, two additional cooling fans were mounted around the sprayer. An unstable Venturi effect driven solvent flow, due to overheating of the ESI sprayer, was avoided and the system was stabilized at higher flow rates and higher temperatures. This enabled placing the ATS4-G4P transducer stage directly in front of the source fixation levers. A minimum capillary length of $25 \mathrm{~cm}$ was required with these modifications (Figure S-3B). For this closer configuration, the GS2 gas lines of the source heaters had to be fixed at a lower position (Figure 2) to avoid contact with the source plate carrier of the ATS-G4P instrument, which was now moving above the source heaters before and after each scan run.

The maximum throughput of the internal flowmeter of the 6500+ QTRAP at a GS1 setting of 90 psi was around $11 \mathrm{~L} / \mathrm{min}$. Thus, the GS1 setting in the Analyst acquisition software was set to zero and its tubing on the sprayer flange was removed. The external flowmeter was then attached directly on the probe flange and the maximum throughput along this supply line was evaluated. Thereby, the nitrogen flow through the ESI sprayer unit was raised to $\sim 19 \mathrm{~L} / \mathrm{min}$ at $300{ }^{\circ} \mathrm{C}$ operating temperature. This was high enough to observe a significant increase of the Venturi effect and thus, of the carrier liquid flow rate. Further improvements can be made by direct modification of the source probe and electrode regarding gas flow optimization.

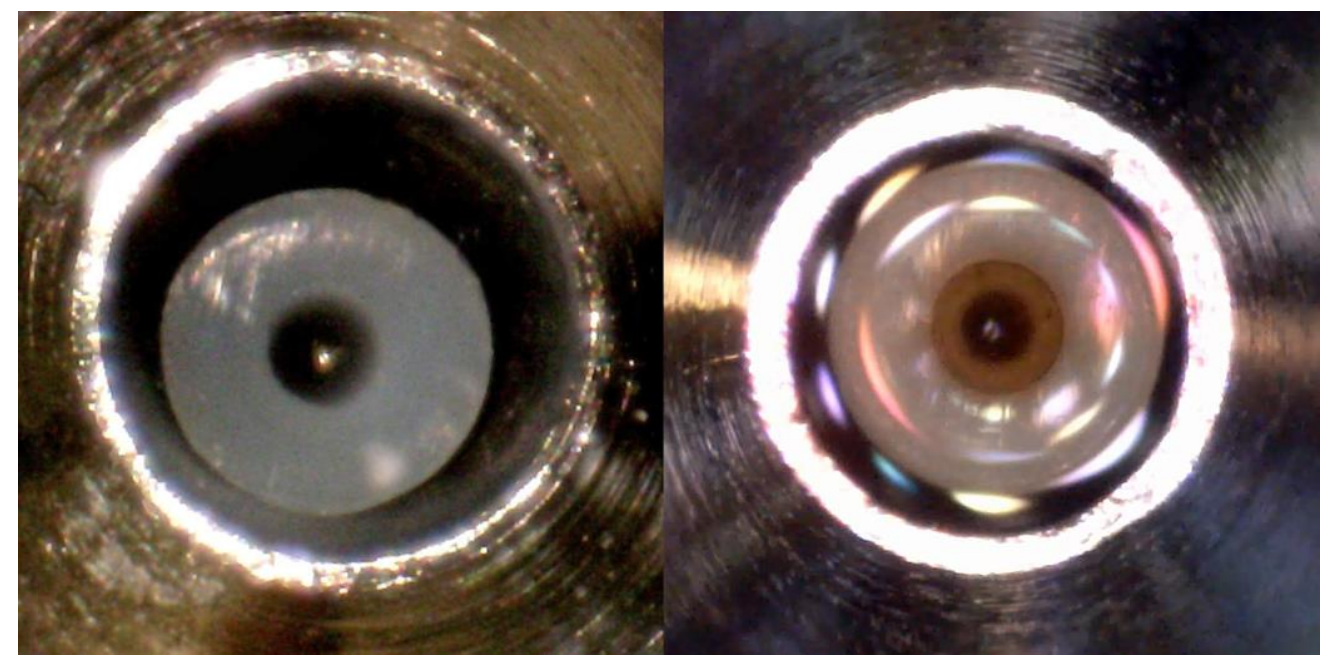

Figure 3. The original OPI (ID $950 \mu \mathrm{m}$ ) and a 1/32" PEEK capillary mounted $\sim 0.5 \mathrm{~mm}$ inside the probe (left). The modified OPI (ID $900 \mu \mathrm{m}$ ) with capillary centering and a 1/32" PEEKsil capillary mounted just $\sim 0.2 \mathrm{~mm}$ inside the probe to reduce capillary forces and the vortex volume by pushing the meniscus outside the probe (right). In the modified probe, the carrier liquid surface tension was visualized by the light refraction. In both probes, the center of the vortex can be seen as a white dot in the center of the image. Please note that the capillary diameters seem to vary due to different refraction.

\subsection{Transfer capillaries and electrodes}

Since the length of the Turbo V ESI electrode was $20.4 \mathrm{~cm}$, the Optiflow Turbo V was used with its $8 \mathrm{~cm}$ electrode. The $\sim 12 \mathrm{~cm}$ decrease in total length between OPI and the tip of the electrode, where the Venturi effect takes place, was crucial for the overall performance. An important detail was the direct crimping of the PEEKsil capillary on the ESI electrode to reduce overall dead volume or the risk of any void volume in capillary fittings. A bad junction increases the backpressure, and thus, the efficiency of the Venturi effect is dramatically reduced. In order to reach the most efficient Venturi effect possible, the positioning of the electrode inside the source probe was evaluated by visual inspection of the liquid vortex stability by the microscope camera (Figure 3 and Video S-1) and the MS baseline stability. Depending on the GS1 gas flow, the maximum Venturi effect can be found at slightly difference electrode protrusions $(0.3-0.5 \mathrm{~mm})$. For GS1 at $19 \mathrm{~L} / \mathrm{min}$, we found the best position at a protrusion of $0.5 \mathrm{~mm}$ out of the source probe, whereas a shorter protrusion was suitable for lower GS1 gas flows. 
Table 1. Influence of the transfer capillary length between the OPI and ESI electrode on the DXM peak parameters. 10 serial injections of $2.5 \mathrm{~nL}$ of $10 \mathrm{nM}$ DXM were performed at a nebulizer flow of 88 psi to determine the $\% R S D$ of peak areas and the peak performance.

\begin{tabular}{ccccc}
\hline $\begin{array}{c}\text { Capillary } \\
\text { length }[\mathrm{cm}]\end{array}$ & $\begin{array}{c}\text { Carrier flow } \\
{[\mu \mathrm{L} / \mathrm{min}]}\end{array}$ & $\begin{array}{c}\% R S D \text { peak area } \\
(n=10)[\mathrm{cps}]\end{array}$ & $\begin{array}{c}\text { Mean peak width at } \\
50 \%(n=10)[\mathrm{ms}]\end{array}$ & $\begin{array}{c}\text { Mean peak width at } \\
5 \%(n=10)[\mathrm{ms}]\end{array}$ \\
\hline 60 & 220 & $6.5 \%$ & 470 & 1070 \\
50 & 220 & $4.9 \%$ & 530 & 1170 \\
40 & 320 & $4.6 \%$ & 360 & 780 \\
35 & 400 & $5.6 \%$ & 320 & 730 \\
30 & 420 & $4.2 \%$ & 270 & 650 \\
25 & 500 & $5.4 \%$ & 260 & 560 \\
\hline
\end{tabular}

In order to achieve a stable liquid vortex at high flow rates $(500-1,000 \mathrm{~mL} / \mathrm{min})$, the positioning and quality of the capillary end on the OPI side was most important. A smooth and orthogonal cut of both kinds of capillary materials (PEEK and PEEKsil) was achieved by using a capillary cutter providing the turning of the capillary against a static razor blade. This was a prerequisite for a stable vortex at high flowrate. PEEK and PEEKsil capillaries are often bent, so that thermal straightening was necessary to obtain a well centered capillary inside the OPI equipped with the centering device. The centered capillary was forming a symmetrical vortex, while a bent capillary will form an asymmetric vortex with different flow rates, leading to unsymmetrical peaks at high low rates.

The influence of the capillary length on the maximum possible carrier flow was investigated for PEEKsil capillaries of $25-60 \mathrm{~cm}$ and their suitable flow rates of $220-500 \mu \mathrm{L} / \mathrm{min}$ using acidified methanol ( $0.1 \%$ FA). The peak area of each 10 injections of $2.5 \mathrm{~nL}$ of $10 \mathrm{nM} \mathrm{DXM}$ was evaluated. Consistent mean \%RSD of 4.2-6.5\% were determined, whereas significant decrease for the mean peak width at 50\% peak height and at 5\% peak height were determined (Table 1). Reducing the capillary length from $50 \mathrm{~cm}$ to $25 \mathrm{~cm}$ reduced the peak width to a half (peak width at $50 \%$ peak height from $530 \mathrm{~ms}$ to $269 \mathrm{~ms}$ and at $5 \%$ peak height from $1170 \mathrm{~ms}$ to $560 \mathrm{~ms}$ ). Thus, a capillary length of $25 \mathrm{~cm}$ was used for the high flow rate experiments.

\subsection{The OPI holder}

A new probe holder (Figure 1A) was mounted instead of the original rotatable target plate holder equipped with a plate adapter for the OPI (Figure S-3). Using the final aluminium design of the probe holder, the construction was rigid enough to reduce the probe to the source plate distance from over $5 \mathrm{~mm}$ to only approximately $1 \mathrm{~mm}$. Deviations in port positioning were avoided by this fixed design and the lowered port height was beneficial to also capture droplets with a suboptimal trajectory. Thus, reproducibility and precision of the system were improved.

In order to use this probe holder in combination with a short $25 \mathrm{~cm}$ capillary, it was necessary to modify the ATS-G4P position database to enable different mechanic movements. The softwarebased activation of movements intended to rotate the removed target plate carrier by its mechanic rotation mechanism moved against a metal pin cannot be deactivated. Thus, these positions were set above the microscope camera. Likewise, the "loading position" of the target plate, intended to move the target plate to the very front in the middle of the instrument, was relocated above the waste port of the OPI. The "target plate" position was fixed to the position intended for the upper left well of a 384-well plate. This position was adjusted and calibrated to be precisely above the acoustic transducer. A MS-based fine tuning was then performed to evaluate the peak area and peak shape depending on the OPI position at slightly varied $x$ - and $y$-directions. After modifying all these positions, the OPI (Figure 1A) was moving just in a small area above the waste port 
(Figure 1C), the microscope camera (B), above the acoustic transducer (Figure 1G) and back to the waste port. The safe standby position of the OPI above the waste port was not only useful for purging the probe, but also during source standby to avoid carrier liquid dripping into the ATS-G4P. For leakage of the carrier liquid or of the coupling liquid for the acoustic unit, two liquid sensors were installed at the bottom of the ATS-G4P (Figure 1F) and were connected to its emergency shut off circuit. These modifications were crucial for the intended implementation into an advanced integrated system. Analogous to the changes of the target plate positions, the loading, barcode reading and standby position of the source plate were relocated to the far right-hand side (Figure 1H) below the relocated barcode scanner (Figure 1I). This enabled an easy access for a manual plate exchange or a dedicated automated plate feeding system.

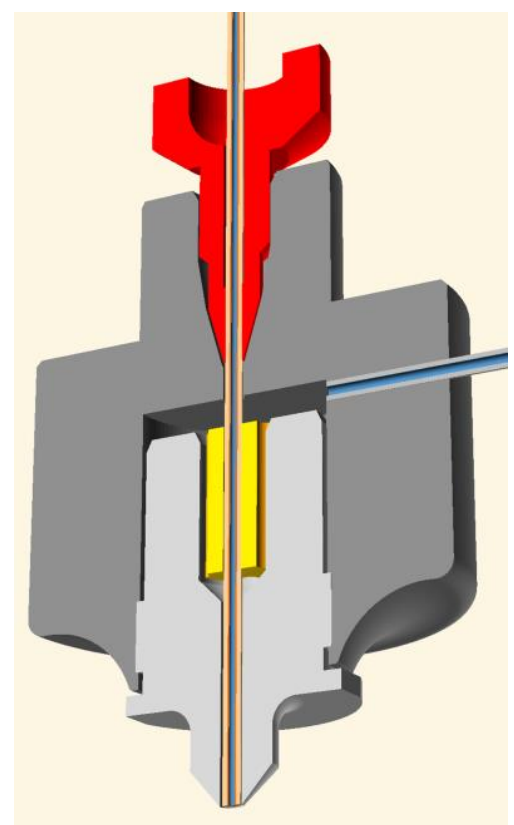

Figure 4. Illustration of the modified OPI. The PEEKsil capillary was vertically mounted by the fitting (red), through the metal centering sleeve (yellow) down to the tip of the probe. The carrier liquid enters the probe on the right side, flushes along the centering sleeve and along the capillary to the tip of the probe.

\subsection{OPI modifications}

The probe main body was used as previously described ${ }^{[18]}$ and was equipped with a $950 \mu \mathrm{m}$ wide probe tip for first experiment (Figure 3, left). Subsequent a $900 \mu \mathrm{m}$ wide probe tip was manufactured to reduce the gap between the probe tip wall and the capillary (Figure 3, right). This new probe tip was equipped with the centering device (Figure 4 and S-7) and allowed to mount the final PEEKsil capillary in the center of the tip with a slight retraction of $\sim 0.2 \mathrm{~mm}$ inside the port. Depending on the flow rate and composition of the carrier liquid, this capillary position enabled to keep the meniscus of the carrier liquid outside the probe. This reduced the capillary forces that have to be compensated by the suction produced by the Venturi effect of the ESI source. Thus, the highest flow rates were only possible with the right capillary position and the respective carrier liquid.

The positioning of the capillary inside the probe, the visualization of the carrier liquid flow and the inspection of the vortex stability were performed with the integrated microscope camera (Figure 1B). This was an important tool to adjust the carrier liquid flow, the electrode protrusion and GS1 gas flow for a stable liquid vortex at high flow rates (Figure 3 and Video S-1). The actual flow rate limit were the probe will overflow was around $0.2-0.3 \mathrm{~mL} / \mathrm{min}$ higher than the flow rate for a visually stable vortex resulting in a stable MS baseline and homogeneous MS signals. 


\subsection{Carrier liquid pumps}

Two systems were tested for precise carrier liquid delivery at low flow variation. The performance of the Agilent 1290 Infinity II versus the HNP microgear pump (in controlled flow and steered flow mode) was evaluated for each 30 injections of $2.5 \mathrm{~nL} 10 \mathrm{nM}$ DXM at a carrier flow of $400 \mu \mathrm{L} / \mathrm{min}$ (capillary length of $35 \mathrm{~cm}$ ) using acidified methanol $(0.1 \% \mathrm{FA})$. All parameters were quite similar between the two systems (flow variation: $0.8 \%-1.9 \%$; peak width at 5\%: 0.70-0.72 s; $\% R S D$ of peak width: $1.4-2.6 \%$; \%RSD of peak area: $4.8-5.4 \%$ ) but the microgear pump had a slightly higher flow variation. This variation was visible as slight fluctuations of the liquid vortex. Nevertheless, signal stability and peak performance was the best for the controlled flow of the microgear pump. The Agilent Infinity II binary pump on the other hand gave a very stable vortex and the capability of online mixing, a subsequent carrier liquid heater (LC column heater) or changing between liquids during operation. The microgear pump in combination with a degasser was a suitable solution for the low-pressure carrier liquid delivery in this integrated design with compact footprint in comparison to a high-pressure LC-pump.

Table 2. Instrument settings and performance data for 50 serial injections of $1 \mathrm{~nL} 100 \mathrm{nM}$ DXM at sampling rates of $1 \mathrm{~Hz}$ and $3 \mathrm{~Hz}$. For $6 \mathrm{~Hz}$ sampling, $100 \mathrm{nM}$ DXM or $100 \mathrm{nM}$ D3-DEX were alternatingly injected $(1 \mathrm{~nL})$ from two wells resulting in $2 \times 576$ injections. To include variations for scanning different wells, the $6 \mathrm{~Hz}$ setup was also evaluated for scanning a triplicate of a completely filled 384-well plate (four injections out of 1152 failed).

\begin{tabular}{lcccc}
\hline & $1 \mathrm{~Hz}$ Setup & 3 Hz Setup & $6 \mathrm{~Hz}$ Setup & $6 \mathrm{~Hz}$ Setup \\
\hline Flow [ $\mu \mathrm{L} / \mathrm{min}]$ & 650 & 1000 & 1000 & 1000 \\
Carrier liquid ratio $(\mathrm{ACN} / \mathrm{MeOH})$ & $0: 100$ & $78: 22$ & $78: 22$ & $78: 22$ \\
Inject. delay [s] & 1.00 & 0.18 & - & - \\
No. of injections $(x)$ & 50 & 50 & $1152[\mathrm{a}]$ & $1148[\mathrm{~b}]$ \\
Nebulizer flow [L/min] & 17 & 19 & 19 & 19 \\
MS dwell time [ms] & 80 & 20 & 15 & 15 \\
Mean $\Delta_{\text {peak max. }}(n=x)[\mathrm{ms}]$ & $1150 \pm 2.0 \%$ & $343 \pm 4.0 \%$ & $329 \pm 3.8 \%$ & $335 \pm 4.4 \%$ \\
$\% R S D$ of peak area $(n=x)$ & $4.5 \%$ & $4.0 \%$ & $7.7 \% / 7.5 \%$ & $8.2 \% / 6.8 \%$ \\
Mean peak width at $50 \%(n=x)[\mathrm{ms}]$ & $209 \pm 10.9 \%$ & $110 \pm 7.3 \%$ & $105 \pm 11.7 \%$ & $107 \pm 10.0 \%$ \\
Mean peak width at 5\% $(n=x)[\mathrm{ms}]$ & $535 \pm 5.5 \%$ & $239 \pm 3.7 \%$ & $237 \pm 5.5 \%$ & $236 \pm 5.8 \%$ \\
Total scan time [s] & 57 & 17 & $3 \times 63[\mathrm{a}]$ & $3 \times 64[\mathrm{~b}]$ \\
\hline
\end{tabular}

[a] Two compounds out of two wells were alternatingly injected

[b] Two compounds out of 384 alternating substance wells were injected

\subsection{Carrier liquid}

The carrier liquid had to meet the following criteria as the OPI is a system open to atmospheric pressure and the Venturi effect of the ESI source was the only force to pull the liquid into the electrode. The viscosity of the liquid has the greatest influence on the maximum carrier liquid flow for a stable Venturi effect. As different carrier liquids are available, the most important requirement was the suitability of the carrier liquid for ESI ionization.

While acidified methanol was used as a carrier liquid for $1 \mathrm{~Hz}$ experiments, suitable for different intended applications, acetonitrile was used for the $3 \mathrm{~Hz}$ and $6 \mathrm{~Hz}$ experiments. The viscosity of acetonitrile was lowered by mixing it with acidified methanol $(78: 22, V / V)$ to obtain the minimum viscosity at this ratio. To enable even higher flowrates, the carrier liquid temperature was regulated between $25-60{ }^{\circ} \mathrm{C}$. The influence of the temperature on viscosity versus increasing diffusion and thus lower peak performance was evaluated regarding the maximum possible flowrate in the OPI 
as well as the signal peak width. An optimum performance was reached at a carrier liquid temperature of $40^{\circ} \mathrm{C}$.

\subsection{Sub-second sampling rate}

The performance on fast sampling rates was evaluated in $1 \mathrm{~Hz}$ (Figure S-9) and $3 \mathrm{~Hz}$ mode

(Figure 5) for 50 injections of each $1 \mathrm{~nL}$ of a $100 \mathrm{nM}$ DXM solution. For the " $6 \mathrm{~Hz}$ " mode (Figure 6) 1152 injections were performed alternatingly with DXM or D3-DEX solution.

For acidified methanol as carrier liquid, the maximum flow rate forming a stable vortex was $650 \mu \mathrm{L} / \mathrm{min}$. In comparison, acetonitrile mixed with acidified methanol (78:22, V/V) enabled a maximum flow rate of $1000 \mu \mathrm{L} / \mathrm{min}$ and a stable vortex at a nebulizer gas flow (GS1) of $19 \mathrm{~L} / \mathrm{min}$. The " $1 \mathrm{~Hz}$ " mode was performed at an injection delay of $1 \mathrm{~s}$ resulting in a mean peak distance of $1150 \mathrm{~ms}(n=50), \% R S D$ of the peak area of $4.5 \%(n=50)$, mean peak width at $5 \%$ height of $535 \mathrm{~ms} \pm 5.5 \%(n=50)$ and a total scan time of $57 \mathrm{~s}$ for 50 injections. The " $3 \mathrm{~Hz}$ " mode was performed at an injection delay of $0.18 \mathrm{~s}$ resulting in a mean peak distance of $343 \mathrm{~ms}(n=50)$, $\% R S D$ of the peak area of $4.0 \%(n=50)$, mean peak width at $5 \%$ height of $239 \mathrm{~ms} \pm 3.7 \%$ $(n=50)$, mean FWHM of $110 \mathrm{~ms} \pm 7.3 \%$ and a total scan time of $17 \mathrm{~s}$ for 50 injections.

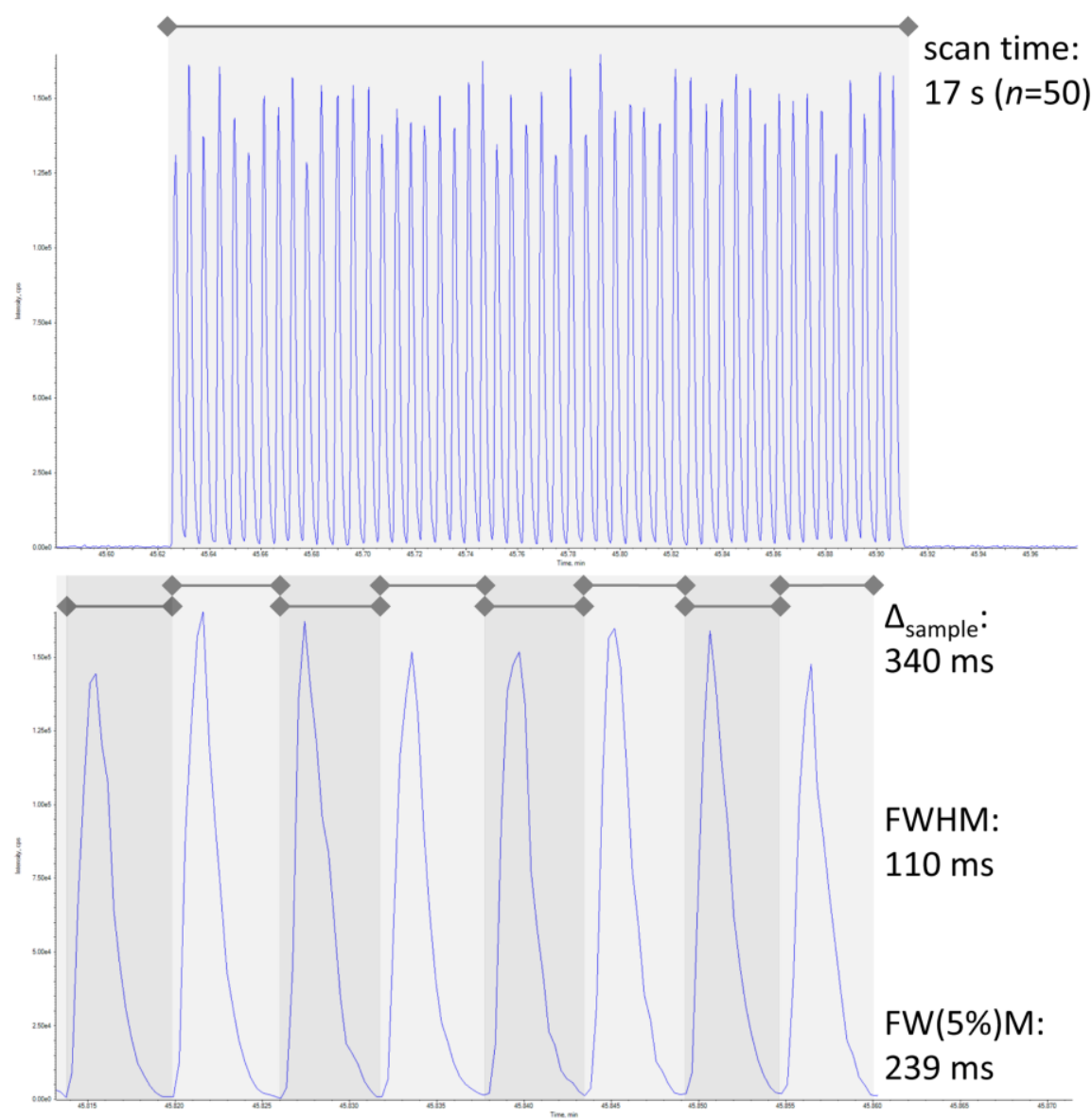

Figure 5. Performance mode for ADE-OPI-ESI-MS at $3 \mathrm{~Hz}$. The MRM scan of 50 injections (each $1 \mathrm{~nL}$ of $100 \mathrm{nM}$ DXM) within $17 \mathrm{~s}$ (top) using acidified acetonitrile-methanol (78/22, V/V) as carrier liquid resulted in homogeneous signal peaks ( $\% R D=4.0 \%, n=50)$ with a mean full width at 5\% peak height of $239 \mathrm{~ms}$ revealed good performance and FWHM of $110 \mathrm{~ms}$ (bottom).

The "6 Hz" mode was performed without an intermittent delay and reduced the delay between each injection to just the time need for positioning and acoustic injection. Also the source plate stage movement acceleration and speed was increased to reach overall injection durations of $165 \mathrm{~ms}$ (Figure 6). For each of the two compounds, the mean peak distance was found to be $329 \mathrm{~ms}(\% R S D$ of $3.8 \%, n=576)$, the $\% R S D$ of peak areas to be $7.7 \%$ and $7.5 \%$ (each $n=576$ ), 
mean peak width at $5 \%$ height of $237 \mathrm{~ms} \pm 5.5 \%(n=1152)$, mean FWHM of $105 \mathrm{~ms} \pm 11.7 \%$ $(n=1152)$ and a total scan time of $63 \mathrm{~s}$ for 384 injections from two adjacent wells. To account for variations in scanning different wells, this configuration was also evaluated for scanning a completely filled 384-well plate (Video S-2). For a triplicated scan, just four injections out of 1152 injections failed in the first cycle. For each of the two compounds, the mean peak-to-peak distance was $335 \mathrm{~ms}(n=573$ and $n=575), \% R S D$ of the peak area of $8.2 \%$ and $6.8 \%(n=573$ and $n=575)$, mean peak width at $5 \%$ height of $236 \mathrm{~ms} \pm 5.8 \% \quad(n=1148)$, mean FWHM of $107 \mathrm{~ms} \pm 10.0 \%(n=1148)$ and a total scan time of $64 \mathrm{~s}$ per 384 -well plate. The demonstrated low peak area deviations and an overall \%RSD of $3.8 \%$ for peak positions (correlating to the injection timing) from both $6 \mathrm{~Hz}$ experiments showed no significant variations and display the reproducible and precise injection and ionization performance of ADE-OPI-ESI-MS at highthroughput (Table 2).

\subsection{System stress and endurance test}

A major benefit of AEMS was the capability to inject samples with no or minor sample preparation as the dilution of the nanoliter-droplet in the carrier liquid reduced ion suppression in comparison to microliter-injections of classic ESI methods. To simulate ion suppression and OPI contamination with residues from assay buffers and plasma components, an OPI stress test was performed (Figure S-10). 720 alternating injections of $2.5 \mathrm{~nL} 100 \mathrm{mM}$ DXM in $0.1 \%$ FA (90\%), CYP buffer (90\%), or dog plasma (90\%), each spaced by solvent blanks $(0.1 \%$ FA), revealed a signal suppression of $51 \%$ for CYP buffer and $73 \%$ for dog plasma. The signal stability of $100 \mathrm{nM}$ DXM was $\% R S D=5.6 \%(n=120)$ in $0.1 \% \mathrm{FA}, \% R S D=5.7 \%(n=120)$ in CYP buffer, and $\% R S D=6.7 \%(n=120)$ in dog plasma. the peak performance declined and the transfer capillary was blocked too much for a stable OPI operation mode. Cleaning with $0.01 \mathrm{NH}_{3}$ was performed successfully after each experiment (Figure S-11A/B). Images of the OPI after 720 injection showed major residues inside the transfer capillary (Figure S-11C/D) that caused the OPI instability after 1100 injections. A simple sample preparation step by $1: 1$ dilution with $0.1 \%$ FA reduced the amount of residues dramatically (Figure S-11E/F).

An endurance test was performed for 25,000 injections of $100 \mathrm{nM}$ in DXM in the HTS endurance buffer, a mix of typically used HTS assay components. Signal stability, peak performance and contaminations of the OPI were investigated to simulate a fraction of an HTS campaign. After $\sim 25,000$ injections the visual inspection of the OPI showed just minor contaminations and no urgent need for a cleaning step (Figure S-12A). Also the signal quality was not influenced and the very first signals in combination with a clean OPI (Figure S-12A, left) were similar to the last signals after $\sim 25,000$ injections with a slightly contaminated OPI (Figure S-12A, right). Data evaluation of the chronogram of the first $\sim 10,000$ injections with removed solvent blanks ( 2 after each plate row and 4 after each plate set) and three removed outliers. These outliers had a $>30 \%$ higher peak area as the mean peak area and were caused by a too intense acoustic ejection. This data set revealed a stable injection performance with $\% R S D=8.5 \%(n=10,557)$ (Figure S-12B) and a constant peak performance of $\mathrm{FWHM}=177 \mathrm{~ms} \pm 8.5 \%$ and $\mathrm{FW} 5 \% \mathrm{M}=374 \mathrm{~ms} \pm 6.4 \%$ (Figure S-12C).

Both experiments revealed the need for the automated cleaning of the OPI and the flushing of the transfer capillary to maintain best performance of the system for complex matrices. During each plate exchange, a time window of 10-30 s of OPI inactivity can be used for this service step.

All experiments were performed without any internal standard correction and demonstrate a high signal stability and suitability for high-throughput applications. Nevertheless, the routine use of isotopic-labeled internal standards will improve the quantitative performance and correct any deviations from the acoustic dispensing and ion suppression for sensible sampling liquids or critical target compounds. 


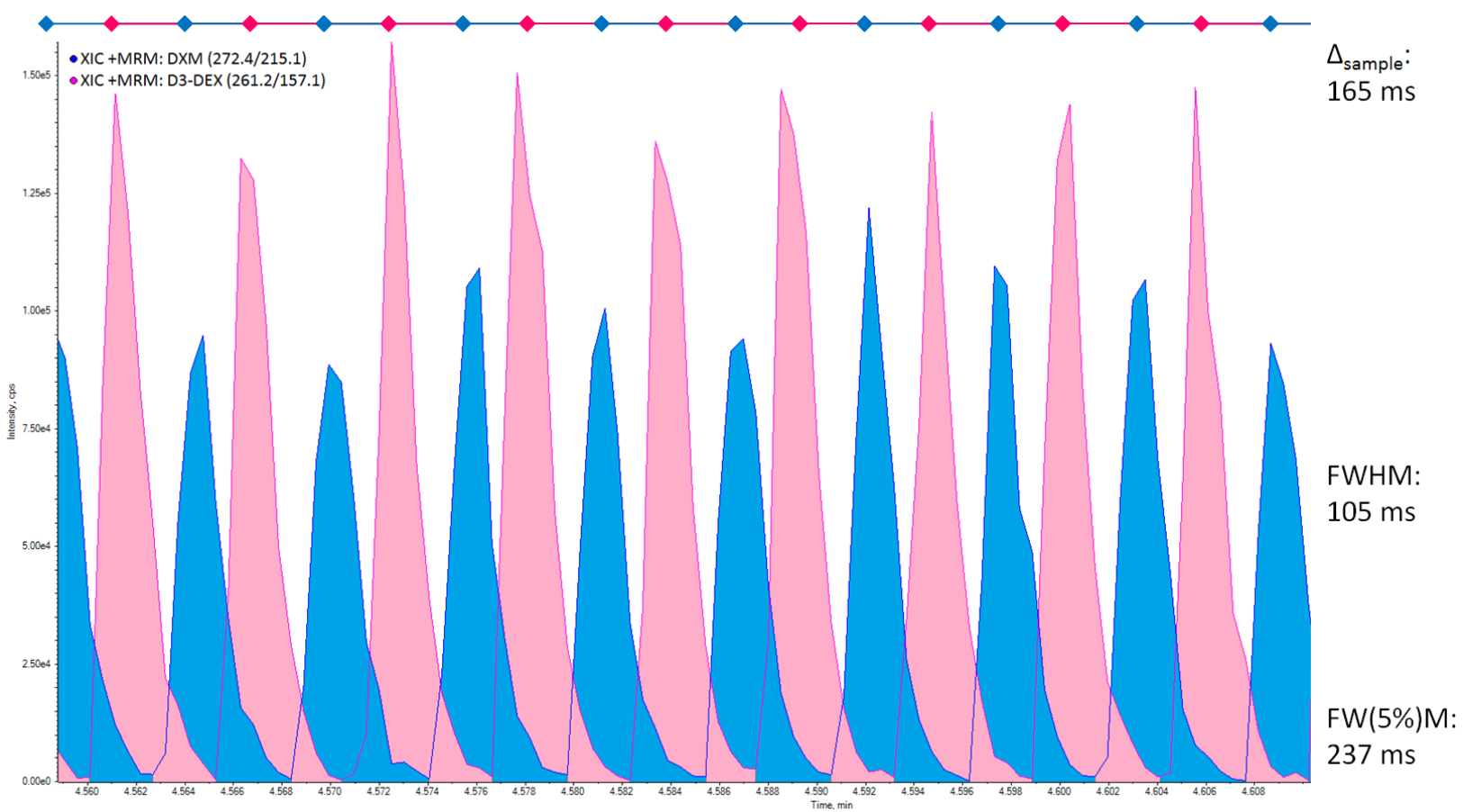

Figure 6. Chronogram of ADE-OPI-ESI-MS at $6 \mathrm{~Hz}$. The scan of 1152 injections of alternating

\section{Conclusion}

408 Based on the modifications of an ATS-G4P acoustic droplet emission system interfaced to a 6500+ QTRAP mass spectrometer with a modified Optiflow Turbo V ion source, the hyphenation of ADE-OPI to ESI-MS was investigated towards its potential as a precise and robust HT screening system. Various modifications and additional equipment resulted in a system that pushes the speed limit of ESI-MS to $3 \mathrm{~Hz}$ for sampling and detection with baseline separation of each sample. This contactless sample injection method provided more than a 7.5 -fold reduction in analysis time compared to the fastest ESI-based systems currently available (Rapid-Fire operated in BLAZE mode at $0.4 \mathrm{~Hz}$ and I-DOT-OPSI at $0.2 \mathrm{~Hz})^{[10,28]}$ and was even faster than the AMI performance for separated peaks of $2.2 \mathrm{~Hz}^{[16]}$ and the maximum HT screening throughput of MALDI at $2.5 \mathrm{~Hz}^{[4]}$

Due to the ultrafast sample injection speed and MS detection rate in the low millisecond-range, even faster sampling rates of $6 \mathrm{~Hz}$ were possible by multiplexing different MRM transitions for different compounds. At lower sampling rates, even unprocessed samples with high matrix loads (e.g. plasma, cell lysate) influencing the peak performance are no hindrance for a fast and contactless sample injection at sample consumption in the low nanoliter-range. The main advantage of this ESI technique is to skip or drastically reduce sample preparation without the risk of high levels of ion suppression, to maintain physiological conditions before sample injection and to even push the speed-limiting step back to the respective assay or sampling technique.

Next steps for AEMS systems are the automation regarding data evaluation, well-plate loading and the final integration in a fully automated and integrated analysis system for various label-free assays at high process stability. 


\section{Supporting Information}

430 Additional supporting information including the experimental section may be found at the publisher's website. Figure S-1 is an illustration of the 3D-model for modifications on the ATSG4P. Figure S-2 displays the ATS-G4P frontend before and after modifications prior to cutting the baseplate. Figure S-3 illustrates the distance and necessary capillary length between the OPI and the ESI electrode. Figure S-4 is an illustration of the complete modified ADE-OPI system. Figure S-5 shows the right side of the ATS-G4P with installed gear pump and degasser components. Figure S-6 is an illustration of the OPI and ESI electrode dimensions and of the carrier liquid flow. Figure S-7 shows the capillary centering device front and the rear side after mounting in the OPI tip. The ATS-G4P on a circular saw quite after cutting the front of the baseplate is shown in Figure S-8. The $1 \mathrm{~Hz}$ standard mode for ADE-OPI-ESI-MS using methanol as carrier liquid is presented in Figure S-9. The OPI stress test and the respective images of the OPI during the stress test are displayed in Figures S-10 and S-11. Results of an endurance test of up to 25,000 injections are presented in Figure S-12. Video S-1 shows the carrier liquid flow inside the OPI at 140x magnification and Video S-2 shows the instrument injecting at $6 \mathrm{~Hz}$ and the recorded MS data of two transitions.

\section{Acknowledgements}

446 We greatly acknowledge all involved coworkers from SCIEX, especially Bryce Young, David Cox, and Jay Corr for their support with instrumentation, troubleshooting, and the inspiring knowledge transfer. We also gratefully acknowledge the fantastic support from our ISEE colleagues of Boehringer Ingelheim, namely the teams of Wolfgang Jörg and Hubert Reiter for their technical support on the several modifications as well as extensions. We also thank David 
452

453

454

455

456

457

458

459

460

461

462

463

464

465

466

467

468

469

470

471

472

473

474

475

476

477

478

479

480

481

482

483

484

485

486

487

488

489

490

491

492

493

494

495

496

497

\section{References}

[1] P. Gribbon, A. Sewing, Drug Discov. Today 2003, 8(22), 1035-1043.

[2] C. Haslam, J. Hellicar, A. Dunn, et al., J. Biomol. Screen. 2016, 21(2), 176-186.

[3] M. Winter, T. Bretschneider, C. Kleiner, et al., SLAS Discov. 2018, 23(6), 561-573.

[4] M. Winter, R. Ries, C. Kleiner, et al., SLAS Technol. 2019, 24(2), 209-221.

[5] R. P. Simon, M. Winter, C. Kleiner, et al., SLAS Discov. 2020, 25(4), 372-383.

[6] T. R. Covey, B. B. Schneider, H. Javaheri, et al. in Electrospray and MALDI Mass Spectrometry: Fundamentals, Instrumentation, Practicalities, and Biological Applications, 2nd ed. (Ed.: R. B. Cole), Hoboken, New Jersey: John Wiley, 2010, pp. 441-490.

[7] A. H. Luippold, T. Arnhold, W. Jörg, B. Krüger, R. D. Süssmuth, J. Biomol. Screen. 2011, 16(3), 370-377.

[8] A. H. Luippold, T. Arnhold, W. Jörg, R. D. Süssmuth, Int. J. Mass Spectrom. 2010, 296(13), 1-9.

[9] X. Wu, J. Wang, L. Tan, et al., J. Biomol. Screen. 2012, 17(6), 761-772.

[10] S. E. Hutchinson, M. V. Leveridge, M. L. Heathcote, et al., J. Biomol. Screen. 2012, 17(1), 39-48.

[11] T. Bretschneider, C. Ozbal, M. Holstein, et al., SLAS Technol. 2019, 24(4), 386-393.

[12] M. Jonas, W. A. LaMarr, C. Ozbal, Comb. Chem. High Throughput Screen. 2009, 12(8), 752-759.

[13] P. T. Rye, L. E. Frick, C. C. Ozbal, W. A. LaMarr, J. Biomol. Screen. 2011, 16(10), 11861195.

[14] T. Bretschneider, A. H. Luippold, H. Romig, et al., SLAS Discov. 2017, 22(4), 425-432.

[15] E. Vanderporten, L. Frick, R. Turincio, P. Thana, W. Lamarr, Y. Liu, Anal. Biochem. 2013, 441(2), 115-122.

[16] I. Sinclair, R. Stearns, S. Pringle, et al., J. Lab. Autom. 2016, 21(1), 19-26.

[17] I. Sinclair, M. Bachman, D. Addison, et al., Anal. Chem. 2019, 91(6), 3790-3794.

[18] H. Zhang, C. Liu, W. Hua, et al., 2020, doi:10.1101/2020.01.28.923938.

[19] K. G. Asano, M. J. Ford, B. A. Tomkins, G. J. Van Berkel, Rapid Commun. Mass Spectrom. 2005, 19(16), 2305-2312.

[20] M. J. Ford, G. J. Van Berkel, Rapid Commun. Mass Spectrom. 2004, 18(12), 1303-1309.

[21] G. J. Van Berkel, A. D. Sanchez, J. M. Quirke, Anal. Chem. 2002, 74(24), 6216-6223.

[22] G. J. Van Berkel, V. Kertesz, R. C. King, Anal. Chem. 2009, 81(16), 7096-7101.

[23] G. E. Morlock, J. Liq. Chromatogr. Relat. Technol. 2014, 37(20), 2892-2914.

[24] T. T. Häbe, G. E. Morlock, Rapid Commun. Mass Spectrom. 2020, 34, e8631.

[25] G. A. Gómez-Ríos, C. Liu, M. Tascon, et al., Anal. Chem. 2017, 89(7), 3805-3809.

[26] G. J. Van Berkel, V. Kertesz, Rapid Commun. Mass Spectrom. 2017, 31(3), 281-291.

[27] G. J. Van Berkel, V. Kertesz, Rapid Commun. Mass Spectrom. 2015, 29(19), 1749-1756.

[28] G. J. Van Berkel, V. Kertesz, M. Orcutt, A. Bentley, J. Glick, J. Flarakos, Anal. Chem. 2017, 89(22), 12578-12586.

[29] C. Liu, G. A. Gómez-Ríos, B. B. Schneider, et al., Anal. Chim. Acta 2017, 991, 89-94.

[30] G. J, Van Berkel, V. Kertesz, H. Boeltz, Bioanalysis 2017, 9(21), 1667-1679.

[31] O. S. Ovchinnikova, D. Bhandari, M. Lorenz, G. J. Van Berkel, Rapid Commun. Mass Spectrom. 2014, 28(15), 1665-1673.

\section{ORCHID}

Tim Häbe: https://orcid.org/0000-0001-8329-4857

Chang Liu: https://orcid.org/0000-0003-0508-4357 
499 A contactless sample injection technique, faster than any current ESI or MALDI based 500 technique, directly out of 384-well plates was demonstrated. The optimized hardware and 501 method setup resulted in high-throughput performance and enables the further implementation 502 in a fully automated platform for ESI-MS-based high-throughput screening.

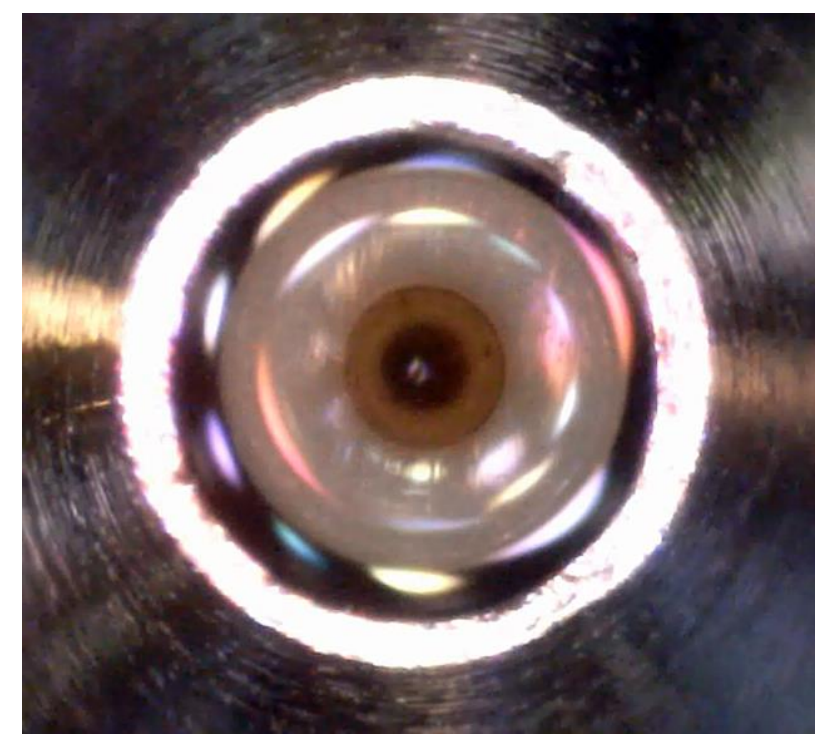

\section{Keywords}

505 Acoustic droplet ejection, analytical methods, liquid vortex capture probe, mass

506 spectrometry, open port interface 